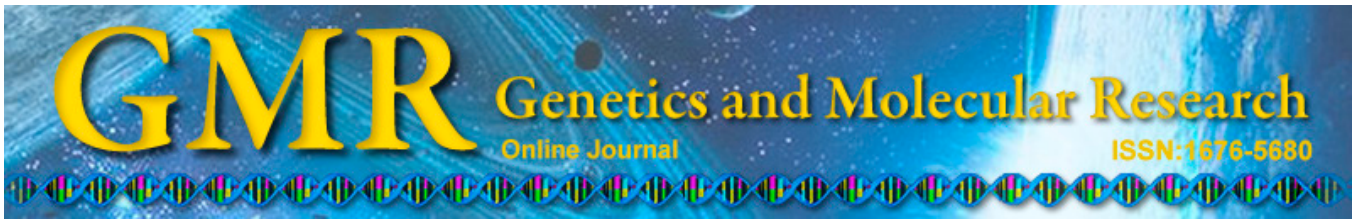

\title{
Isolation and characterization of novel polymorphic microsatellite markers for Lutjanus erythropterus
}

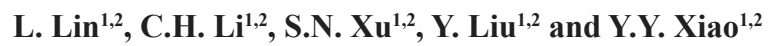

${ }^{1}$ Key Laboratory for Exploitation \& Utilization of Marine Fisheries Resources in the South China Sea, Ministry of Agriculture, Guangzhou, China

${ }^{2}$ South China Sea Fisheries Research Institute, Chinese Academy of Fishery Sciences, Guangzhou, China

Corresponding author: C.H. Li

E-mail: scslch@vip.163.com

Genet. Mol. Res. 14 (3): 10944-10947 (2015)

Received January 14, 2015

Accepted May 28, 2015

Published September 21, 2015

DOI http://dx.doi.org/10.4238/2015.September.21.5

\begin{abstract}
We isolated and characterized 22 polymorphic microsatellite loci in the crimson snapper (Lutjanus erythropterus) using a $(\mathrm{GT})_{13}$-enriched genomic library. We found three to 15 alleles per locus, with a mean of 6.68 . The observed and expected heterozygosities ranged from 0.087 to 0.978 and from 0.125 to 0.904 , respectively, with averages of 0.576 and 0.650 , respectively. Only three loci showed significant deviation from the Hardy-Weinberg equilibrium after Bonferroni correction. Four loci showed evidence for null alleles. These markers will be useful for analyzing the population genetic structure and gene flow of $L$. erythropterus.
\end{abstract}

Key words: Lutjanus erythropterus; Crimson snapper; Microsatellite marker; Population structure 


\section{INTRODUCTION}

Crimson snapper, Lutjanus erythropterus, is a pelagic fish that is widely distributed throughout the Indian Ocean and the subtropical and tropical parts of the western Pacific Ocean. This species is an important fish resource in China. Recent data collected within the past three decades indicate that the stock density and yield of L. erythropterus have dramatically declined (Guo et al., 2011). The conservation and sustainable utilization of L. erythropterus resources in Chinese coastal waters has drawn the attention of regulatory authorities. An evaluation of the population genetic structure is critical for making informed conservation and management decisions for this species. Microsatellites are important tools for examining genetic diversity and population genetic structure. Here, we report the development of 22 novel polymorphic microsatellite markers for L. erythropterus that will improve the ability to detect population genetic structure and gene flow of $L$. erythropterus.

\section{MATERIAL AND METHODS}

Fifty fishes were collected from the sea around Sanya, China. The samples were preserved at $-20^{\circ} \mathrm{C}$ until DNA extraction. A dinucleotide-enriched genomic library was constructed following a previously published method (Ma and Chen, 2009). In brief, DNA was extracted from muscle tissue and digested with the restriction enzyme MseI (New England Biolabs, USA). The digested DNA fragments were ligated to MseI adaptor pair (5'-TACTCAGGACTCAT-3'/5'-GACGATGAGTCCTGAG-3'). The ligated products were then pre-amplified in a $25-\mu \mathrm{L}$ reaction mixture using the adapter specific primer (5'-GATGAGTCCTGAGTAA-3') to verify successful ligation and increase DNA concentration. A biotin-labeled (GT) ${ }_{13}$ probe was applied to hybridize with the pre-amplification products. The hybridized complexes were captured using streptavidin-coated magnetic beads (Promega, USA) and then eluted, forming a library of GT-rich DNA fragments.

Subsequently, the library DNA was amplified using the adapter-specific primer and ligated into pMD 18-T plasmid vectors (TaKaRa, Japan), and Escherichia coli DH5 $\alpha$ competent cells were transformed with these plasmid vectors. The positive clones were randomly sequenced using an ABI Prism 3730 automated DNA sequencer (Applied Biosystems, USA). Microsatellite repeats were found in 62 sequenced clones. Primer pairs were designed for 53 of the microsatellite loci with suitable flanking regions for amplification by polymerase chain reaction (PCR) using the Primer Premier 5 software (PREMIER Biosoft International, USA).

The designed primer pairs were evaluated using 50 individuals of L. erythropterus. PCR was performed on a Veriti Thermal Cycler (Applied Biosystems, USA) in a total reaction volume of $25 \mu \mathrm{L}$, containing $0.4 \mu \mathrm{M}$ primer (each), $0.2 \mathrm{mM}$ dNTP (each), 1X PCR buffer, 2 $\mathrm{mM} \mathrm{MgCl}, 1 \mathrm{U}$ Taq polymerase (TaKaRa, Japan), and 10-100 ng DNA. The amplification profile consisted of an initial denaturing step of $94^{\circ} \mathrm{C}$ for $5 \mathrm{~min}, 35$ cycles of $45 \mathrm{~s}$ at $94^{\circ} \mathrm{C}, 50 \mathrm{~s}$ at the locus-specific annealing temperature, and $45 \mathrm{~s}$ at $72^{\circ} \mathrm{C}$, followed by a final step of $72^{\circ} \mathrm{C}$ for $10 \mathrm{~min}$. The PCR products were separated on $6 \%$ denaturing polyacrylamide gel and visualized by silver staining. Observed and expected values of heterozygosity as well as deviation from the Hardy-Weinberg equilibrium were calculated using GENEPOP 4.0 (Rousset, 2007). Null allele frequencies (Brookfield, 1996) were estimated using MICRO-CHECKER 2.2.3 (Van Oosterhout et al., 2004). All P values were corrected for multiple testing using Bonferroni correction (Rice, 1989). 


\section{RESULTS AND DISCUSSION}

Twenty-two of 53 loci were cleanly amplified and shown to be polymorphic. The number of alleles per locus ranged from three to 15 with an average of 6.68 (Table 1). The observed and expected heterozygosities ranged from 0.087 to 0.978 and from 0.125 to 0.904 , respectively, with averages of 0.576 and 0.650 , respectively (Table 1). Only loci LE76, LE88, and LE107 showed significant deviation from Hardy-Weinberg proportions after Bonferroni correction $(\mathrm{P}<0.0023)$. Four loci (LE76, LE88, LE95, and LE107) showed evidence of null alleles (estimated null allele frequency $>5 \%$ ).

\begin{tabular}{|c|c|c|c|c|c|c|c|c|c|}
\hline Locus & Primer sequence $\left(5^{\prime}-3^{\prime}\right)$ & Repeat motif & $\begin{array}{l}\mathrm{Ta} \\
\left({ }^{\circ} \mathrm{C}\right)\end{array}$ & $\begin{array}{l}\text { Allele size } \\
\text { range (bp) }\end{array}$ & $N_{\mathrm{A}}$ & $H_{\mathrm{O}}$ & $H_{\mathrm{E}}$ & $\mathrm{P}_{\mathrm{HW}}$ & $\begin{array}{l}\text { GenBank } \\
\text { accession No. }\end{array}$ \\
\hline LE58 & $\begin{array}{l}\text { F: TGTGAACTTTCTTTTGGAT } \\
\text { R: AGTAACTATAAGCCCTCGA }\end{array}$ & $(\mathrm{CA})_{18}$ & 50 & $330-380$ & 9 & 0.756 & 0.871 & 0.004 & KC006902 \\
\hline LE60 & $\begin{array}{l}\text { F: AAGCACTGTTTACAGCAG } \\
\text { R: GAGAAAAGAAGAAAGGTTA }\end{array}$ & $(\mathrm{AC})_{13} \mathrm{~N}(\mathrm{CA})_{12} \mathrm{~N}(\mathrm{CAC})_{5}$ & 47 & $250-350$ & 9 & 0.848 & 0.820 & 0.667 & KC006903 \\
\hline LE76 & $\begin{array}{l}\text { F: CTTGGAGGAAGGTGGGTT } \\
\text { R: TCAAGCACTCGGAGCAAA }\end{array}$ & $(\mathrm{TG})_{8} \mathrm{~N}(\mathrm{GT})_{5}$ & 50 & $200-220$ & 7 & 0.476 & 0.625 & 0.000 & KC006904 \\
\hline LE77 & $\begin{array}{l}\text { F: CAGGACGGTTCGCATTAC } \\
\text { R: AGGCTGGCAGGTAGACAA }\end{array}$ & $(\mathrm{AC})_{9}$ & 50 & $250-300$ & 8 & 0.773 & 0.802 & 0.675 & KC006905 \\
\hline LE80 & $\begin{array}{l}\text { F: CAGCACCCATTCAAGCAA } \\
\text { R: CGACAGACTGGCGAGAAA }\end{array}$ & $(\mathrm{CA})_{7}$ & 50 & $220-250$ & 3 & 0.087 & 0.125 & 0.016 & KC006906 \\
\hline LE $88^{* \dagger}$ & $\begin{array}{l}\text { F: ATTTACCAGGGTTCACAG } \\
\text { R: CTCTTTATTTGGGTAGCG }\end{array}$ & $(\mathrm{CA})_{22} \mathrm{~A}(\mathrm{AC})_{9}$ & 47 & $260-320$ & 9 & 0.391 & 0.778 & 0.000 & KC006907 \\
\hline LE95* & $\begin{array}{l}\text { F: AAAAGCTCCACATATCCACT } \\
\text { R: TATCACTCCGCTAAGAACG }\end{array}$ & $(\mathrm{CA})_{10}$ & 58 & $180-220$ & 8 & 0.643 & 0.766 & 0.003 & KC006908 \\
\hline LE99 & $\begin{array}{l}\text { F: GAAATTTAGCATGGAATACAA } \\
\text { R: TCTCAATGGAAACTTCACTG }\end{array}$ & $(\mathrm{CA})_{5} \mathrm{CC}(\mathrm{CA})_{10} \mathrm{~N}(\mathrm{CT})_{8}$ & 47 & $270-290$ & 3 & 0.341 & 0.380 & 0.330 & KC006909 \\
\hline LE100 & $\begin{array}{l}\text { F: TACCAGAAAGGCCCATTAGA } \\
\text { R: CAGACCAGACGAGGAGACG }\end{array}$ & $(\mathrm{TG})_{11}$ & 58 & $170-200$ & 4 & 0.200 & 0.223 & 0.500 & KC006910 \\
\hline LE103 & $\begin{array}{l}\text { F: TACTCTGGTGGCGGAGCAATG } \\
\text { R: TCTGGGCTGATGTTCGTGGAT }\end{array}$ & $(\mathrm{AC})_{15}$ & 45 & $210-240$ & 5 & 0.756 & 0.686 & 0.121 & KC006911 \\
\hline LE $107^{* \dagger}$ & $\begin{array}{l}\text { F: GAGATGTGAGGTGCTGTG } \\
\text { R: GTCTATTCTGACGGGAGC }\end{array}$ & $(\mathrm{GT})_{10}$ & 55 & $200-230$ & 4 & 0.222 & 0.586 & 0.000 & KC006912 \\
\hline LE116 & $\begin{array}{l}\text { F: TATGGAGACTTGCTTGTGGTC } \\
\text { R: CTACTTTGTCCTGGGTAATGC }\end{array}$ & $(\mathrm{CA})_{10}$ & 45 & $270-310$ & 6 & 0.651 & 0.711 & 0.014 & KC006913 \\
\hline LE151 & $\begin{array}{l}\text { F: TCCAAGGATTACGTGTATG } \\
\text { R: GAGGTGTAAGGAAAGCAGA }\end{array}$ & $(\mathrm{AC})_{9} \mathrm{C}(\mathrm{CA})_{5} \mathrm{AC}(\mathrm{CA}) 5$ & 52 & $200-220$ & 3 & 0.556 & 0.646 & 0.493 & KC006914 \\
\hline LE161 & $\begin{array}{l}\text { F: ACGAGTAAACAGCCCTTCT } \\
\text { R: AGTCCTGAGTAATAGTGAGCC }\end{array}$ & $(\mathrm{AC})_{15}$ & 45 & $240-300$ & 11 & 0.743 & 0.828 & 0.003 & KC006915 \\
\hline LE170 & $\begin{array}{l}\text { F: TCTGCCCTCAGACCACAG } \\
\text { R: TTCCCAAGTGCTCAAGTGT }\end{array}$ & $(\mathrm{AC})_{10} \mathrm{AA}(\mathrm{CA})_{9}$ & 47 & $190-210$ & 5 & 0.512 & 0.542 & 0.130 & KC006916 \\
\hline LE172 & $\begin{array}{l}\text { F: CCCTACCCATGATGACGA } \\
\text { R: GACTTGATCTGCCCCTGA }\end{array}$ & $(\mathrm{CA})_{19}$ & 47 & $240-300$ & 10 & 0.978 & 0.882 & 0.097 & KC006917 \\
\hline LE177 & $\begin{array}{l}\text { F: GGCTGTCGGCATAAGAAGTGT } \\
\text { R: GCTGGGTGCTGATGTGACTAA }\end{array}$ & $(\mathrm{CA})_{6}$ & 47 & $190-230$ & 9 & 0.696 & 0.810 & 0.007 & KC006918 \\
\hline LE181 & $\begin{array}{l}\text { F: GCACGCTTGTAGTTATGGAC } \\
\text { R: ATTAGAGGGTAAGTGGGAGG }\end{array}$ & $(\mathrm{AC})_{23}$ & 55 & $300-400$ & 5 & 0.773 & 0.679 & 0.578 & КC006919 \\
\hline LE183 & $\begin{array}{l}\text { F: CTACTTTGTCCTGGGTAATGC } \\
\text { R: TATGGAGACTTGCTTGTGGTC }\end{array}$ & $(\mathrm{TG})_{10}$ & 53 & $270-310$ & 6 & 0.682 & 0.717 & 0.158 & КС006920 \\
\hline LE187 & $\begin{array}{l}\text { F: GCTTTTAGCCCATTTTATTC } \\
\text { R: TACAGTTGTGCCCTGAGATT }\end{array}$ & $(\mathrm{CA})_{6}$ & 45 & $250-310$ & 3 & 0.087 & 0.125 & 0.016 & KC006921 \\
\hline LE189 & $\begin{array}{l}\text { F: CCCTACCCATGATGACGA } \\
\text { R: GACTTGATCTGCCCCTGA }\end{array}$ & $(\mathrm{CA})_{19}$ & 52 & $240-300$ & 15 & 0.913 & 0.904 & 0.107 & KC006922 \\
\hline LE190 & $\begin{array}{l}\text { F: AAGGAGCGAGCGTGTTCT } \\
\text { R: TGTGGGCAGGTATTTGAG }\end{array}$ & $(\mathrm{GT})_{5} \mathrm{~N}(\mathrm{TG})_{5}$ & 47 & $242-270$ & 5 & 0.587 & 0.789 & 0.030 & KC006923 \\
\hline
\end{tabular}

Ta $=$ optimized annealing temperature; $N_{\mathrm{A}}=$ number of alleles; $H_{\mathrm{O}}=$ observed heterozygosity; $H_{\mathrm{E}}=$ expected heterozygosity; $\mathrm{P}_{\mathrm{HW}}=$ Hardy-Weinberg probability test, *locus may harbor null alleles (estimated null allele frequency $>5 \%$ ); ’ locus deviated from Hardy-Weinberg proportions (adjusted P value $<0.0023$ ). 


\section{ACKNOWLEDGMENTS}

Research supported by the Special Fund for Agro-Scientific Research in the Public Interest (Grant \#201403008) and the China Postdoctoral Science Foundation (Grant \#2013M531882).

\section{REFERENCES}

Brookfield JFY (1996). A simple new method for estimating null allele frequency from heterozygote deficiency. Mol. Ecol. 5: 453-455.

Guo YS, Wang ZD, Xie ZQ and Liu CW (2011). Isolation and genetic diversity analysis of microsatellite DNA in Lutjanus erythropterus. J. Guangdong Ocean Univ. 31: 13-16.

Ma HY and Chen SL (2009). Isolation and characterization of 31 polymorphic microsatellite markers in barfin flounder (Verasper moseri) and the cross-species amplification in spotted halibut (Verasper moseri). Conserv. Genet. 10: 1591-1595.

Rice WR (1989). Analyzing tables of statistical tests. Evolution 43: 223-225.

Rousset F (2007). Genepop '007: a complete reimplementation of the genepop software for Windows and Linux. Mol. Ecol. Resour. 8: 103-106.

Van Oosterhout C, Hutchinson WF, Wills DPM and Shipley P (2004). MICRO-CHECKER: software for identifying and correcting genotyping errors in microsatellite data. Mol. Ecol. Notes 4: 535-538. 